\title{
AUXINAS ENDÓGENAS, AIA-OXIDASA Y ENRAIZAMIENTO EN Vigna radiata L. Wilczek INDUCIDO POR AUXINA EXÓGENA LIBRE Y CONJUGADA
}

\author{
ENDOGENOUS AUXINS, IAA-OXIDASE AND ROOTING IN Vigna radiata L. Wilczek INDUCED BY \\ EXOGENOUS FREE AND CONJUGATED AUXIN
}

\author{
César M. Flores Ortiz*, Apolinar Cabañas-Cabrera, Ignacio Peñalosa Castro, Rafael E. Quintanar Zúñiga, \\ Josefina Vázquez Medrano y Martha A. Urzúa Meza
}

Laboratorio de Fisiología Vegetal, Unidad de Biología y Prototipos, Facultad de Estudios Superiores Iztacala, Universidad Nacional Autónoma de México. Av de los Barrios 1. 54090, Los Reyes Iztacala, Edo. México. Tel. (0155) 5623-1131, Fax (0155) 5623-1225.

*Autor para correspondencia (cmflores@servidor.unam.mx)

\section{RESUMEN}

La regeneración de plantas con fenotipos de interés tiene una etapa crítica en el enraizamiento adventicio, que mejora con la aplicación de auxina. La disponibilidad de auxina libre juega un papel importante en el desarrollo vegetal; la conjugación-desconjugación y la degradación oxidativa influyen en ella. No se encontraron antecedentes que evaluaran si la aplicación de un conjugado artificial de auxinas promueve cambios en la auxina endógena en el curso del enraizamiento. En este trabajo se comparó el efecto del conjugado indol-3-acetil-p-nitrofenil éster (IAP), con el de las auxinas libres, ácido indol-3-acético (AIA) y ácido indol-3-butírico (AIB), en los niveles endógenos de AIA y AIB en tres secciones de plántulas, en la actividad AIA-oxidasa (AIA-ox), y en el enraizamiento adventicio durante 6 d, en Vigna radiata L. Wilczek. El nivel de AIA endógeno se incrementó significativamente en el día tres en todos los tratamientos, especialmente en la sección apical de la plántula, con excepción del tratamiento con IAP en el que sólo aumentó ligeramente. La actividad de AIA-ox se incrementó en el día tres y gradualmente disminuyó hacia el sexto día. El IAP provocó el mayor enraizamiento y el menor correspondió al AIA.

Palabras clave: Vigna radiata, auxinas, conjugados, enraizamiento.

\section{SUMMARY}

Plant regeneration of special phenotypes has a critical phase in adventitious rooting, which is promoted by auxin treatment. Avalilability of free auxin plays an important role in plant development; auxin conjugation-deconjugation and oxidative degradation have an influence on it. No report was found in which synthetic auxin conjugates had been evaluated for promoting changes in endogenous auxin during rooting. This study analized the auxin content in plant cuttings after administration of indole-3-acetil- $p$ nitrophenyl ester (IAP) (a synthetic auxin-conjugate). The effect of exogenous auxins indole-3-acetic acid (IAA), indole-3-butyric acid (IBA) and IAP on endogenous levels of IAA and IBA, in three different seedling sections and on IAA-oxidase activity, were compared during the first $6 \mathrm{~d}$ of adventitious root formation in Vigna radiata L. Wilczeck. Endogenous IAA level increased significantly on the 3rd day of rooting induction in all treatments, specially in the apical section, with the exception of IAP which only induced a slight increase. IAA-ox activity reached the highest rate at day 3 and then became reduced gradually to day 6 . The highest adventitious rooting was obtained with IAP, and the lowest with IAA.

Index words: Vigna radiata, auxins, conjugates, rooting.

\section{INTRODUCCIÓN}

La formación de raíces adventicias es promovida por auxinas como ácido indol-3-acético (AIA) y ácido indol-3butírico (AIB) (Woodward y Bartel, 2005; Davies, 2004). Los factores que controlan los niveles de auxina libre son: las peroxidasas con actividad de AIA-oxidasa (AIA-ox) (McDonald, 2003; Metaxas et al., 2004); el transporte polar (Friml, 2003; Benjamins y Scheres, 2008); y la conjugación, que es la formación de ésteres o amidas entre auxinas y azúcares o aminoácidos (Bartel et al., 2001; Woodward y Bartel, 2005).

La aplicación exógena de auxinas afecta la biosíntesis apical y el transporte basípeto de auxinas endógenas (Teale et al., 2006), de modo que los niveles de las auxinas libres permanecen altos durante la fase de inducción del enraizamiento y decrecen justo al comenzar la fase de iniciación (Gaspar y Hofinger, 1988; Nag et al., 2001). En contraste, las peroxidasas de Clase III (EC 1.11.1.7) (Nag et al., 2001; Qaddoury y Amssa, 2004) muestran baja actividad de AIA-ox durante la inducción, seguida de un aumento durante la iniciación. Además, en esa fase los niveles de conjugados auxínicos se incrementan (Davies, 2004). En consecuencia, la disminución rápida de AIA libre, justo después del comienzo de la fase de iniciación de las raíces adventicias, puede deberse a la mayor 
actividad catalítica de la AIA-ox, y a la formación de conjugados de AIA.

Las auxinas tienen un efecto variable en la inducción de raíces adventicias. El mayor enraizamiento que promueve el AIB se ha atribuido principalmente a tres factores: su capacidad de inducir el movimiento del AIA desde el epicotilo hacia el hipocotilo (Teale et al., 2006); su transformación lenta y continua en AIA (Bartel et al., 2001; Ludwig-Müller, 2000); y su mayor estabilidad química (Gorter, 1961; Nordström et al., 1991).

La función principal de los conjugados de auxina se ha relacionado con la protección de las auxinas a la oxidación enzimática, con su transporte y su almacenamiento (Bartel, 1997; Rampey et al., 2004). La adición a las plantas de conjugados-auxínicos sintéticos que puedan ser convertidos rápidamente a conjugados naturales puede ser una herramienta útil para entender el destino de las auxinas aplicadas exógenamente, lo que pudiera demostrar una mayor eficiencia del compuesto conjugado, como se ha reportado para el AIB-aspartato (Wiesman et al., 1989). El objetivo del presente trabajo fue comparar el efecto de adicionar dos auxinas naturales (AIA y AIB) y el de un conjugado (IAP) sintetizado en el laboratorio para lograr una baja energía de activación, sobre el contenido endógeno de auxinas (AIA y AIB), la actividad de la AIA-ox y el enraizamiento en frijol mungo (Vigna radiata L. Wilczek).

\section{MATERIALES Y MÉTODOS}

Material Vegetal. Setenta gramos de semillas comerciales de frijol mungo se trataron con $100 \mathrm{~mL}$ de $\mathrm{NaClO}$ $4 \%$ durante $30 \mathrm{~min}$, luego puestas a germinar en agua destilada por $24 \mathrm{~h}$ en oscuridad a $26{ }^{\circ} \mathrm{C}$ y después transferidas a una cámara de crecimiento a $26^{\circ} \mathrm{C}$ en la oscuridad y $60 \%$ de humedad relativa, durante $4 \mathrm{~d}$. Se escogieron plántulas con la mayor uniformidad posible en altura, diámetro de hipocotilo y tamaño de cotiledones y hojas. Las plántulas se segmentaron en esquejes que constaron de epicotilo, hojas primarias, cotiledones y $8 \mathrm{~cm}$ de hipocotilo, y éstas fueron el material vegetal del experimento.

Soluciones de tratamiento. Las soluciones de AIA, AIB e IAP $100 \mu \mathrm{M}$ se prepararon en soluciones concentradas $100 \mathrm{X}$, y luego se disolvieron $50 \mu \mathrm{mol}$ de cada compuesto en $2.5 \mathrm{~mL}$ de etanol a $96 \%$, y se llevaron a un volumen final de $5 \mathrm{~mL}$ con agua destilada. Se agregó 1 $\mathrm{mL}$ de cada una de estas soluciones concentradas para preparar $100 \mathrm{~mL}$ de solución de tratamiento.

Ensayo de enraizamiento. Las condiciones para el ensayo de enraizamiento se establecieron de acuerdo con lo propuesto por Pan y Gui (1997), con algunos ajustes. Se distribuyeron al azar 240 esquejes de $12 \mathrm{~cm}$, en ocho grupos de 30 esquejes cada uno. Los ocho grupos se dividieron en dos conjuntos (repeticiones); a cada conjunto se le asignaron los cuatro tratamientos: Testigo (que constó de $2.5 \mathrm{~mL}$ de etanol a $96 \%$ en $100 \mathrm{~mL}$ de agua) y los correspondientes a AIA, AIB, e IAP. Los esquejes se mantuvieron en las soluciones de AIA, AIB e IAP durante 24 $\mathrm{h}$, y se transfirieron después de enjuagarlos con agua destilada, a contenedores de $80 \mathrm{~mL}$ con $50 \mathrm{~mL}$ de agua destilada, que se colocaron en una cámara de crecimiento en oscuridad a $26{ }^{\circ} \mathrm{C}$ y $60 \%$ de humedad relativa, durante 4 d. Se evaluó el enraizamiento adventicio al quinto día del corte, mediante el registro del número de raíces adventicias por hipocotilo, que incluyó tanto primordios como raíces emergidas, en todos los esquejes de cada grupo. Los resultados se examinaron por medio de un análisis de varianza y una prueba de medias de Tukey.

Extracción de auxinas. Se cuantificó el contenido de auxinas al término del primero, tercero y sexto día de tratamiento, con las condiciones de extracción descritas por McDougall y Hillman (1978). Los esquejes se dividieron en tres regiones: epicotilo, la mitad superior y la mitad inferior del hipocotilo. Se homogeneizaron en mortero $5 \mathrm{~g}$ de tejido fresco de cada región, en $15 \mathrm{~mL}$ de metanol mezclado con polivinilpolipirrolidona (PVPP) a una concentración final de $0.1 \%$, durante $10 \mathrm{~min}$, y luego se filtraron a través de ocho capas de gasa. El filtrado se evaporó al vacío y se le adicionaron $10 \mathrm{~mL}$ de $\mathrm{NaHCO}_{3}$ a 2 $\%$. La solución obtenida se extrajo dos veces con $15 \mathrm{~mL}$ de éter dietílico, con eliminación de la fase etérea en cada ocasión, y la fase acuosa recuperada se acidificó con $\mathrm{HCl}$ $2 \mathrm{~N}$. Finalmente, la fracción acuosa acidificada enriquecida de auxinas se extrajo dos veces más con $25 \mathrm{~mL}$ de éter dietílico. El éter se eliminó con vacío y el residuo se resuspendió en $1 \mathrm{~mL}$ de fase móvil.

Condiciones del cromatógrafo. El contenido de auxinas en cada muestra se analizó en un cromatógrafo de líquidos (Hewlett Packard $1100 \AA$ ) provisto con una columna de fase reversa Hypersil ODS, con tamaño de partícula $3 \mu \mathrm{m}, 4.6 \mathrm{~mm}$ de diámetro interno y $60 \mathrm{~mm}$ de longitud (Nordström et al., 1991); se inyectaron $20 \mu \mathrm{L}$ de muestra a un flujo de $0.5 \mathrm{~mL}$ para los primeros $2 \mathrm{~min}$, luego se usó una rampa de 0.5 a $2 \mathrm{~mL}$ de 2 a $8 \mathrm{~min}$. La fase móvil contenía acetonitrilo:ácido acético:agua (10:2:88, v/v). La determinación de auxinas se hizo con un detector de fluorescencia (Perkin-Elmer LS-50B $\AA$ ), a una longitud de onda de excitación de $280 \mathrm{~nm}$ y $340 \mathrm{~nm}$ de emisión. La cuantificación se hizo por comparación con un estándar externo de concentración conocida de cada auxina, con un factor de recuperación de $85 \%$ a través de ensayos de enriquecimiento. 
Actividad de AIA-oxidasa. Un gramo de tejido fresco de esquejes se homogenizó en $1.5 \mathrm{~mL}$ de solución, que contenía PVPP $1 \%$ en Tris-HCl 10 mM, pH 7.6. El extracto se filtró con ocho capas de gasa y se centrifugó a $10000 \times \mathrm{x}$ durante $15 \mathrm{~min}$. El sobrenadante recuperado se empleó para el análisis enzimático. La actividad de AIAox se registró polarográficamente (Kokkinakis y Brooks, 1979). Se adicionó un volumen de extracto que contuviera $100 \mu \mathrm{g}$ de proteína total a $1 \mathrm{~mL}$ de medio de reacción, cuya composición incluyó: 2,4 diclorofenol $120 \mu \mathrm{M}$, $\mathrm{H}_{2} \mathrm{O}_{2} 6 \mu \mathrm{M}, \mathrm{MnCl}_{2} 200 \mu \mathrm{M}$, AIA $300 \mu \mathrm{M}$ y amortiguador de citratos $5 \mathrm{mM}, \mathrm{pH} 4.2$. El consumo de $\mathrm{O}_{2}$ se midió con un electrodo tipo Clark (Hansatech mod. CB1D ${ }^{\circledR}$ ). La proteína se cuantificó de acuerdo con Bradford (1976), con albúmina de suero de bovino como estándar.

\section{RESULTADOS Y DISCUSIÓN}

Ensayo de enraizamiento. La mayor inducción de raíces se obtuvo con el tratamiento con IAP $100 \mu \mathrm{M}$ en el que se registró un promedio de 83.1 raíces por esqueje, lo que representa $340 \%$ de incremento con respecto al testigo (Figura 1). El tratamiento con AIB también estimuló el enraizamiento, pero en menor proporción (264\%). Ambos tratamientos superaron $(\mathrm{P} \leq 05)$ al testigo.

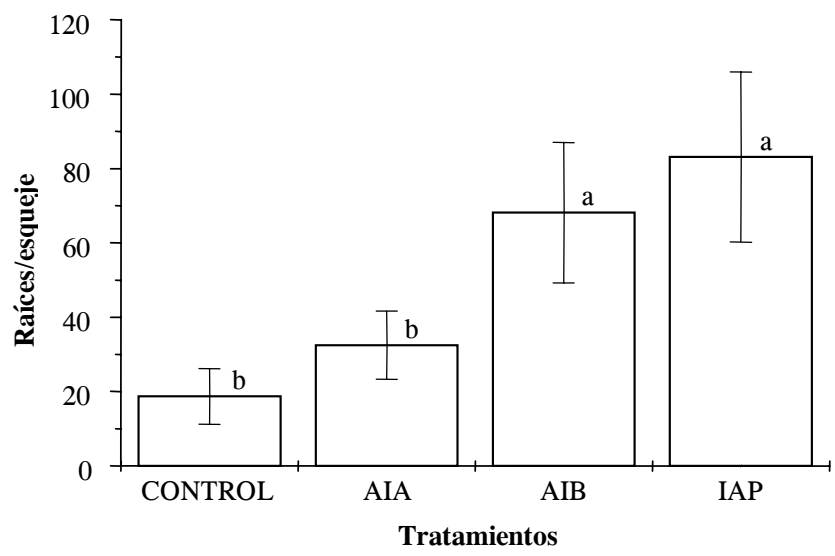

Figura 1. Número de raíces emitidas por los esquejes de Vigna radiata $\mathrm{L}$. Wilczek, $5 \mathrm{~d}$ después de la aplicación de AIA (ácido indolacético), AIB (ácido indolbutírico) e IAP (indolacetato de p-nitrofenil éster). Columnas con la misma letra no son significativamente diferentes (Tukey, 0.05).

Contenido de auxinas. En el primer día los niveles de AIA del testigo alcanzaron su mayor concentración en el hipocotilo, y en todos los tratamientos (incluyendo al testigo) los niveles de AIA fueron prácticamente nulos en la región del epicotilo (Figura 2). En los tres tratamientos con aplicación de auxinas exógenas, el epicotilo mostró un incremento característico en los niveles endógenos de AIA durante la iniciación del enraizamiento (hasta el tercer día), y una disminución durante la emergencia de raí- ces (después del tercer día). El incremento más alto de AIA ocurrió al tercer día en el grupo tratado con AIB; en contraste, el testigo mostró una disminución en AIA, en ambas mitades (superior e inferior) del hipocotilo, a los 3 y $6 \mathrm{~d}$.

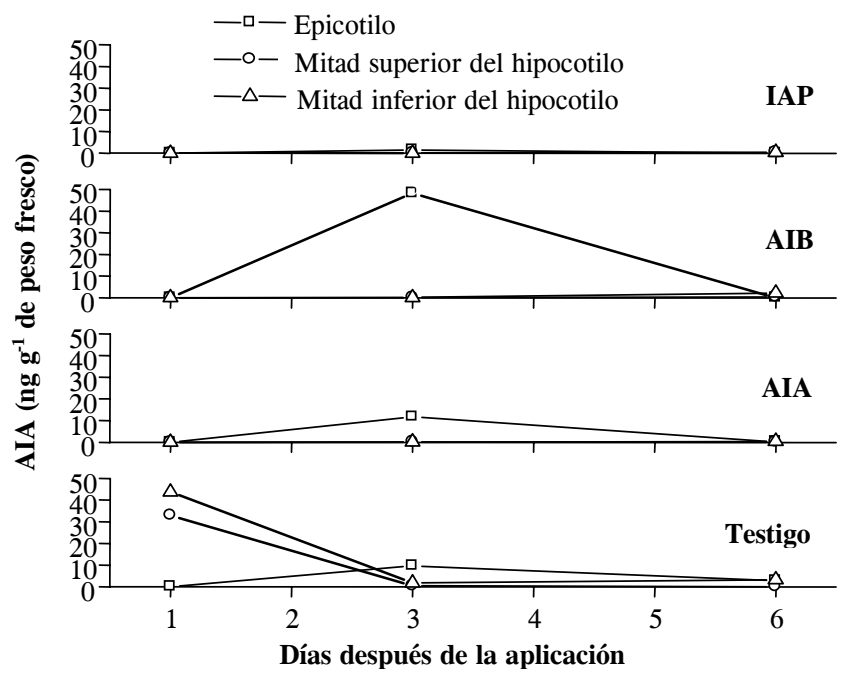

Figura 2. Contenido de AIA (ácido indolacético) en los segmentos de epicotilo, mitad superior e inferior del hipocotilo a 1, 3 y $6 \mathrm{~d}$ después de la aplicación de tratamientos.

En general, los niveles de AIB fueron menores que los de AIA (Figuras 2 y 3). La mayor concentración de AIB se registró en el epicotilo del grupo tratado con AIB, al tercer día (Figura 3). Hubo un ligero incremento de AIB a medida que progresaba el enraizamiento, con la excepción del grupo tratado con AIB que lo incrementó más al tercer día y lo disminuyó drásticamente al sexto. Entre los tres tejidos analizados, el epicotilo generalmente contenía los niveles más altos de AIB.

Los niveles de AIA en las plantas tratadas con IAP fueron menores que en las tratadas con AIA y AIB (Figuras 2 y 3), aunque presentaron una tendencia similar (porque también aumentaron hasta el tercer día y luego disminuyeron hasta el día 6). En este mismo tratamiento con IAP, los niveles de AIB mostraron un incremento hasta alcanzar el más alto valor $(0.54 \mathrm{ng}$ de $\mathrm{AIB} / \mathrm{g}$ de tejido fresco) en el epicotilo, al sexto día. Los niveles de AIB en los tres tejidos analizados durante los tres días de muestreo, fueron menores que los correspondientes a los tratamientos de AIA y AIB.

Actividad de AIA-oxidasa. La actividad de AIA-ox (Figura 4) se incrementó hasta el día 3 y disminuyó hacia el día 6, en los tres tratamientos con auxinas. El valor mayor se registró al tercer día con IAP. En contraste, la actividad menor se encontró en el testigo, al primer día. En general, se observó que en los primeros 3 d la 
actividad de AIA-ox fue mayor en los tratamientos que tuvieron el mayor enraizamiento. En las Figuras 1 y 4 se muestra que todos los tratamientos que promovieron el enraizamiento tuvieron mayor actividad de AIA-ox al tercer día, seguido de un decremento hacia el sexto día, una vez que las raíces habían sido iniciadas. La actividad de AIA-ox en el grupo testigo se incrementó gradualmente hasta el sexto día, cuando ocurrió el inicio del enraizamiento adventicio.

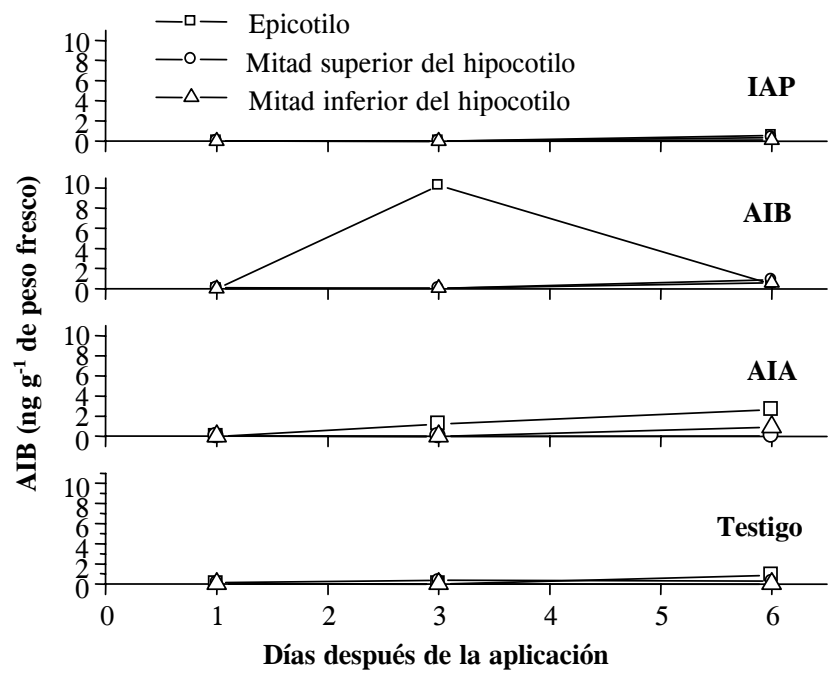

Figura 3. Contenido de AIB (ácido indolbutírico) en los segmentos del epicotilo, mitad superior e inferior del hipocotilo a 1,3 y 6 d después de la aplicación de tratamientos.

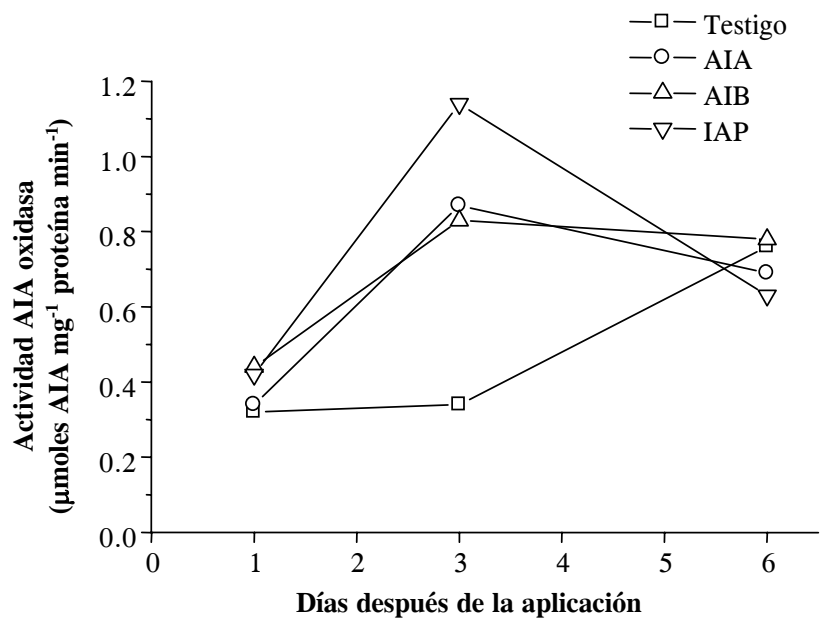

Figura 4. Actividad de AIA-oxidasa en los 1,3 y $6 \mathrm{~d}$ después de la aplicación de tratamientos.

El enraizamiento es resultado de eventos de inducción y de regulación metabólica. Los niveles de AIA encontrados en este trabajo son similares a los cuantificados en estudios previos (Nordström et al., 1991; Pan y Gui, 1997). Los niveles de AIA mostraron una acumulación inicial y un decremento subsecuente, en tanto que la acti- vidad de AIA-ox se incrementó; estos resultados son similares a los consignados por Jarvis y Shaheed (1986) y Nag et al. (2001). El aumento de AIA corresponde al estímulo auxínico requerido para el primer estadio del enraizamiento.

Una posible explicación del incremento en los niveles de AIA observado en los esquejes tratados con AIA, es la incorporación de la auxina exógena añadida cuya absorción pasiva pudo aumentarlos, aunque se ha reportado que la mayor parte de las auxinas aplicadas son transformadas a sus conjugados (Andreae y Wood, 1955; Woodward y Bartel, 2005). De modo que el encontrar niveles altos en su forma libre puede deberse a que la aplicación de auxina alteró una o más de las vías que controlan su concentración.

En cuanto a los niveles de AIA detectados en los esquejes tratados con AIB, aunque la conversión de AIB en AIA es posible (Bartel et al., 2001; Srivastava, 2002), el incremento de AIA en los esquejes fue mayor que la cantidad de AIB añadido, lo que implica un cambio en el control homeostático del AIA en el que pueden participar la síntesis, el transporte, la oxidación y la conjugación. Jarvis y Shaheed (1986) encontraron resultados similares en frijol mungo, y sugirieron que el aumento en la formación de raíces adventicias y en el AIA endógeno asociado con la aplicación de AIB se debe a una mayor translocación basípeta de AIA, En cambio, Ludwig-Müller et al. (2005), al trabajar con Arabidopsis thaliana, sugirieron que la mayor eficiencia del AIB sobre la formación de raíces adventicias se debe a una interacción entre el AIB exógeno y el AIA endógeno, que implica el transporte basípeto de AIA, según lo observado al aplicar TIBA que inhibe el transporte de AIA.

La elevación pronunciada en el AIA puede ser responsable del mayor número de raíces adventicias que se registró en los esquejes tratados con AIB, aunque no se descarta que el AIB por sí mismo actúe directamente como auxina y no a través de su conversión en AIA (LudwigMüller, 2000). Adicionalmente, el incremento de los niveles endógenos de AIA y AIB con el tratamiento de AIB (Figuras 2 y 3), mostró que la acumulación de AIB libre es más lenta y de menor magnitud que la producida por AIA; es decir, las concentraciones de AIB fueron menores que las de AIA, pero tendieron a incrementarse. Estos resultados son similares a los reportados por LudwigMüller et al. (1993).

Weisman et al. (1989) reportaron que después de $24 \mathrm{~h}$ de la aplicación de AIB a esquejes de frijol mungo, menos de $20 \%$ del AIB permaneció en forma libre y poco más de $50 \%$ se conjugó con ácido aspártico o con derivados 
de alto peso molecular, lo que puede explicar en alguna medida los niveles más bajos de AIB libre encontrados en este trabajo. Diversos autores han atribuido la alta capacidad del AIB para inducir el enraizamiento, a su mayor estabilidad química y a su menor oxidación enzimática, comparada con la del AIA (Ludwig-Müller, 2000; Gaspar y Hofinger, 1988). De acuerdo con esto, el nivel bajo del AIB podría no resultar de su degradación oxidativa sino de su rápida conjugación y posiblemente de su baja polaridad, que le produce un movimiento más lento desde la solución hasta el tejido, con respecto al AIA.

En la AIA-ox se observó que la actividad total se incrementó en los primeros 3 d y luego comenzó a disminuir, excepto en esquejes del testigo. Este resultado sugiere que el aumento de actividad es una respuesta a la aplicación de auxina para controlar el exceso de auxina libre, que puede estar regulada por el aumento de la concentración endógena del AIA. En la segunda fase la disminución en la actividad puede deberse al control por retroalimentación negativa, directa o indirecta, ejercida por productos de la actividad enzimática o por una o más señales disparadas por el aumento transitorio inicial de AIA. Una posibilidad interesante es que las peroxidasas del apoplasto se activaran al acidificarse las inmediaciones del tejidodestino de las auxinas, al tomar en cuenta que las auxinas inducen acidificación del apoplasto (Robert et al., 2003), y que existen peroxidasas con actividad de AIA-ox cuyo pH óptimo es de 5 (Johri et al., 2005).

Los resultados aquí obtenidos confirman la reactividad química del IAP, puesto que el compuesto sintetizado es un éster de AIA que requiere menor energía de activación para esterificar el grupo carbonilo. Así, esta molécula podría ser transformada rápidamente a un conjugado de AIA en presencia de agentes nucleofílicos, carbohidratos o aminoácidos, con asistencia de enzimas como la recién evidenciada en extractos de Pisum sativum L., que catalizan su síntesis a partir de aminoácidos como el aspártico, que son los conjugados predominantes en dicotiledóneas (Ostroski y Jakubowska, 2008). Es importante mencionar que el tratamiento con IAP mostró la mayor actividad de AIA-ox, lo que puede estar asociado con el hecho de que los niveles de IAP mantienen un bajo pero constante desprendimiento de auxina libre. Por tanto, el sistema de degradación de AIA recibe una inducción constante por una liberación gradual, a partir de la forma conjugada. Es decir, la degradación lenta podría producir estimulos por un periodo mayor, lo que resultaría en una estimulación continua de enraizamiento adventicio. Es notable que la aplicación del IAP promovió el mayor enraizamiento adventicio sin que fuera acompañado, como ocurrió al aplicar AIB, de un aumento comparable en los niveles endógenos de AIA (el aumento fue tan sólo de $1.47 \mathrm{ng} \mathrm{g}^{-1}$ de tejido fresco).

Este contraste puede ser explicado si, como recientemente sugirieron Walz et al. (2008), las auxinas al conjugarse con proteínas directamente o a partir de un derivado activado, quedan dotadas de un grupo prostético cuya significación puede ir más allá del papel de regulación de los niveles de auxina libre. También se podrían explicar por la presencia de hidrolasas de conjugados auxínicos, como las encontradas en Medicago truncatula (Campanella et $a l ., 2008)$, que de localizarse en las inmediaciones del tejido-destino facilitarían la liberación del AIA a partir de sus conjugados en zonas determinadas. La localización puntual de las hidrolasas determinaría la elevación de AIA en regiones también muy específicas.

\section{CONCLUSIONES}

La aplicación de IAP incrementa en forma significativa la inducción de raíces en esquejes de frijol mungo, en comparación con auxinas libres como el AIA y AIB, sin elevar significativamente las concentraciones de AIA o AIB endógenos. La aplicación exógena de auxinas induce su acumulación endógena, principalmente en la región del epicotílo durante la fase de iniciación del enraizamiento. La actividad de la AIA-oxidasa muestra un incremento en su tasa de actividad al momento de la iniciación del enraizamiento, y disminuye gradualmente con la emergencia de las raíces adventicias.

\section{BIBLIOGRAFÍA}

Andreae W A, N E Good (1955) The formation of indoleacetylaspartic acid in pea seedlings. Plant Physiol. 30:380-382.

Bartel B (1997) Auxin biosynthesis. Annu. Rev. Plant Physiol. Plant Mol. Biol. 48:51-66.

Bartel B S, S LeClere, M Magidin, B K Zolman (2001) Inputs to the active indole-3-acetic acid pool: de novo synthesis, conjugate hydrolysis, and indole-3- butiyric acid $\beta$-oxidation. J. Plant Growth Reg. 20:198-216.

Benjamins R, B Scheres (2008) Auxin: The looping star in plant development. Annu. Rev. Plant Biol. 59:443-465.

Bradford M M (1976) A rapid and sensitive method for the cuantitation of microquantities of protein. Analyt. Biochem. 72:248-253.

Campanella J J, S M Smith, D Leibu, S Wexler, J Ludwig-Müller (2008) The auxin conjugate hydrolase family of Medicago truncatula and their expression during the interaction with two symbionts. J. Plant Growth Reg. 27:26-38.

Davies P J (2004) The plant hormones: their nature, ocurrence and functions. In: Plant Hormones, Biosynthesis, Signal Transduction, Action. P J Davies (ed). Kluwer Acad. Pub. Netherlands. pp:5-6.

Friml J (2003) Auxin transport-shaping the plant. Curr. Opin. Plant Biol. 6:7-12.

Gaspar T, M Hofinger (1988) Auxin metabolism during adventitious rooting. Adv. Plant Sci. Series 2:117-131. 
Gorter C J (1961) Morphogenetic effects of synthetic auxins. In: Encyclopaedia of Plant Physiology, Vol 14. W Ruhland (ed). Springer-Verlag, Berlin. pp:1084-1109.

Jarvis B C, A I Shaheed (1986) Adventitious root formation in relation to the uptake and distribution of supplied auxin. New Phytol. 103:23-31

Johri S, U Jamwal, S Rasool, A Kumar, V Verma, G N Qazi (2005) Purification and characterization of peroxidases from Withania somnifera (AGB 002) and their ability to oxidize IAA. Plant Sci. 169:1014-1021.

Kokkinakis D M, J L Brooks (1979) Hydrogen peroxide mediated of indole-3-acetic acid by tomato peroxidase and molecular oxygene. Plant Physiol. 64:220-223.

Ludwig-Müller J (2000) Indole-3-butyric acid in plant growth and development. Plant Growth Reg. 32:219-230.

Ludwig-Müler J, S Sass, E G Sutter, M Wodner, E Epstein (1993) Indole-3-butyric acid in Arabidopsis thaliana I. Identification and quantification. Plant Growth Reg. 13:179-187.

Ludwig-Müller J, A Vertocnik, C D Town (2005) Analysis of indole3-butyric acid-induced adventitious root formation on Arabidopsis stem segments. J. Exp. Bot. 56:2095-2105.

McDonald M S (2003) Adventitious root formation in woody tissue: peroxidase a predictive marker of root in Betula pendula. In vitro Cell. Develop. Biol. 39:234-236.

McDougall J, J R Hillman (1978) Purification of IAA from shoot tissues of Phaseolus vulgaris and its analysis by GC-MS. J. Exp. Bot. 29:375-386.

Metaxas D J, T D Syros, T Yupsanis, A S Economou (2004) Peroxidases during adventitious rooting in cuttings of Arbutus unedo and Taxus baccata as affected by plant genotype and growth regulator treatment. Plant Growth Reg. 44:257-266.

Nag S, K Saha, M A Choudhuri (2001) Role of auxin and polyamines in adventitious root formation in relation to changes in compounds involved in rooting. J. Plant Growth Reg. 20:182194.

Nordström A C, F Alvarado, L Eliasson (1991) Effect of exogenous indole-3-acetic acid and indole-3-butiric acid on internal lev- els of the respective auxins and their conjugation with aspartic acid during adventitious root formation in pea cuttings. Plant Physiol. 96:856-861.

Ostroski M, A Jakubowska (2008) Identification of enzyme activity that conjugates indole-3-acetic acid to aspartate in immature seeds of pea (Pisum sativum). J. Plant Physiol. 165:564-569.

Pan R, H Gui (1997) Physiological basis of the synergistic effects of IBA and triadimefon of rooting of mung bean hypocotyls. Plant Growth Reg. 22:7-11.

Qaddoury A, M Amssa (2004) Effect of exogenous indole butyric acid on root formation and peroxidase and indole-3-acetic acid oxidase activities and phenolic contents in date palm offshoots. Bot. Bull. Acad. Sin. 45:127-131.

Rampey R A, S LeClere, M Kowalczyck, K Ljung, G Sandberg, B Bartel (2004). A family of auxin-conjugate hydrolases that contributes to free indole-3-acetic acid levels during Arabidopsis germination. Plant Physiol. 135:978-988.

Robert K N, J T P Albrechtová, J Fleig, N Huck, W Michalke, E Wagner, V Speth, G Neuhaus, C Fischer (2003) Plasma membrane $\mathrm{H}^{+}$-ATPase is involved in auxin-mediated cell elongation during wheat embryo development. Plant Physiol. 131:1302-1312.

Srivastava L M (2002) Plant Growth and Development. Hormones and Environment. Academic Press. London, UK. pp:166-167.

Teale W D, I A Paponov, K Palme (2006) Auxin in action: signalling, transport and the control of plant growth and development. Nature Rev. Mol. Cell Biol. 7:847-859.

Walz A, C Seidel, G Rusak, S Park, J D Cohen, J Ludwig-Müller (2008) Heterologous expression of IAP1, a seed protein from bean modified by indole-3-acetic acid, in Arabidopsis thaliana and Medicago truncatula. Planta 227:1047-1061.

Weisman Z, J Riov, E Epstein (1989) Characterization and rooting ability of indole-3-butyric acid conjugates formed during rooting of mung bean cuttings. Plant Physiol. 91:1080-1084.

Woodward A W, B Bartel (2005) Auxin: regulation, action, and interaction. Ann. Bot. 95:707-735. 